CRYSTALLOGRAPHIC COMMUNICATIONS

ISSN 2056-9890

Received 12 July 2016

Accepted 26 July 2016

Edited by $\mathrm{H}$. Stoeckli-Evans, University of Neuchâtel, Switzerland

Keywords: crystal structure; 8-hydroxyquinoline derivative; vinyl; inversion dimer; hydrogen bonding; inversion dimers; $\mathrm{C}-\mathrm{H} \cdots \pi$ interactions.

CCDC reference: 859030

Supporting information: this article has supporting information at journals.iucr.org/e

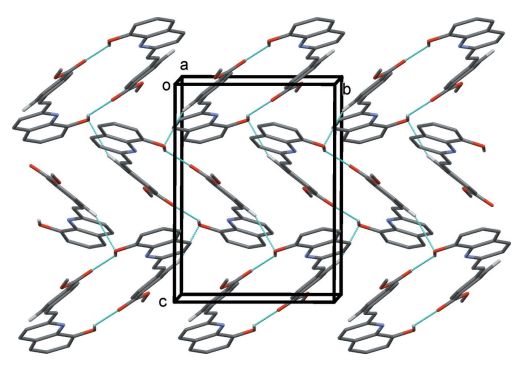

OPEN $\odot$ ACCESS

\section{Crystal structure of methyl (E)-4-[2-(8-hydroxy- quinolin-2-yl)vinyl]benzoate}

\author{
Yu-Xing $\mathrm{Xu}{ }^{\mathrm{a}}$ Wei-Ji Hu ${ }^{\mathrm{a}}$ and Guo-Liang Zhao ${ }^{\mathrm{a}, \mathrm{b} *}$ \\ ${ }^{a}$ College of Chemistry and Life Science, Zhejiang Normal University, Jinhua, Zhejiang 321004, People's Republic of \\ China, and ' XingZhi College, Zhejiang Normal University, Jinhua, Zhejiang 321004, People's Republic of China. \\ *Correspondence e-mail: sky53@zjnu.cn
}

The title compound, $\mathrm{C}_{19} \mathrm{H}_{15} \mathrm{NO}_{3}$, was synthesized by a Perkin reaction of 2-methyl-8-hydroxyquinoline and 4-formyl-2-methylbenzoate in acetic anhydride under a nitrogen atmosphere. The molecule has an $E$ conformation about the $\mathrm{C}=\mathrm{C}$ bond, and the quinoline ring system and the benzene ring are inclined to one another by $29.22(7)^{\circ}$. There is an intramolecular $\mathrm{O}-\mathrm{H} \cdots \mathrm{N}$ hydrogen bond in the 8-hydroxyquinoline moiety. In the crystal, molecules are linked by pairs of $\mathrm{O}-\mathrm{H} \cdots \mathrm{O}$ hydrogen bonds, forming inversion dimers with an $R_{2}^{2}(28)$ ring motif. The dimers are linked by $\mathrm{C}-\mathrm{H} \cdots \mathrm{O}$ hydrogen bonds and $\mathrm{C}-\mathrm{H} \cdots \pi$

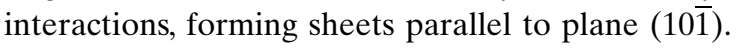

\section{Chemical context}

In recent years, 8-hydroxyquinoline and its derivatives have played an important role in coordination chemistry (Albrecht et al., 2008; Cacciatore et al., 2013), shown to exhibit biological activity (du Moulinet d'Hardemare et al., 2012) and have found various applications in the fields of synthetic chemistry (Song et al., 2006) and organic light-emitting diodes, which have been extensively exploited in the synthesis of luminescent metal complexes (Tang et al., 1987). It is therefore highly desirable to develop new efficient 8-hydroxyquinoline derivatives for use in luminescent metal complexes. In the present work, we report on the synthesis and crystal structure of a new 8-hydroxyquinoline derivative, synthesized by the Perkin reaction of 2-methyl-8-hydroxyquinoline and 4-formyl-2methylbenzoate.<smiles>COC(=O)c1ccc(/C=C/c2ccc3cccc(O)c3n2)cc1</smiles>

\section{Structural commentary}

The molecular structure of the title compound is shown in Fig. 1. It contains an 8-hydroxyquinoline moiety, with an intramolecular $\mathrm{O}-\mathrm{H} \cdots \mathrm{N}$ hydrogen bond (Fig. 1 and Table 1), 


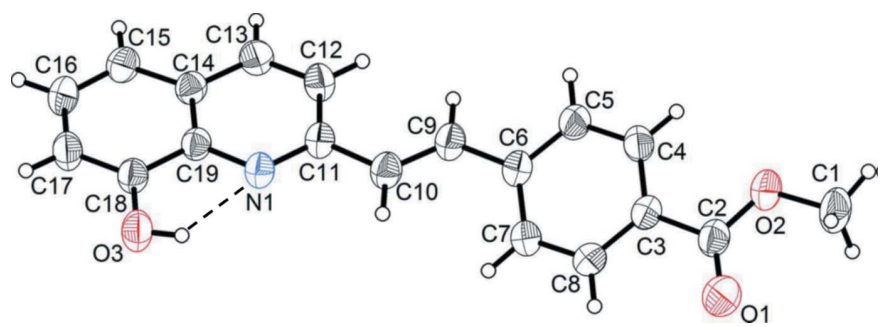

Figure 1

View of the molecular structure of the title compound, showing the atom labelling and $40 \%$ probability displacement ellipsoids. The intramolecular $\mathrm{O}-\mathrm{H} \cdots \mathrm{N}$ hydrogen bond is shown as a dashed line (see Table 1).

and a methylbenzoate unit. They are linked by the $\mathrm{C} 9=\mathrm{C} 10$ bond [1.321 (2) $\AA$ ] with an $E$ conformation. The C11-C10 and C6-C9 bond lengths are 1.463 (2) and 1.466 (2) $\AA$, respectively. These distances are shorter than the standard length of a $\mathrm{C}-\mathrm{C}$ single bond ( $c a 1.5 \AA$ ) because of the conjugate system formed by the $\mathrm{C} 9=\mathrm{C} 10$ bond and the aromatic systems. The quinoline ring system and the benzene ring are inclined to one another by $29.22(7)^{\circ}$.

\section{Supramolecular features}

In the crystal, molecules are linked by pairs of $\mathrm{O}-\mathrm{H} \cdots \mathrm{O}$ hydrogen bonds, forming inversion dimers with an $R_{2}^{2}(28)$ ring motif (Table 1 and Fig. 2). The dimers are linked by $\mathrm{C}-\mathrm{H} \cdots \mathrm{O}$ hydrogen bonds and $\mathrm{C}-\mathrm{H} \cdots \pi$ interactions, forming sheets parallel to $(10 \overline{1})$; see Table 1 and Fig. 3.

\section{Database survey}

A search of the Cambridge Structural Database (CSD, Version 5.37, last update May 2016; Groom et al., 2016) for the substructure 2-styrylquinolin-8-ol gave 17 hits; however, certain of these involve bis(8-hydroxyquinolines) or a (9anthryl) moiety. Three compounds are similar to the title

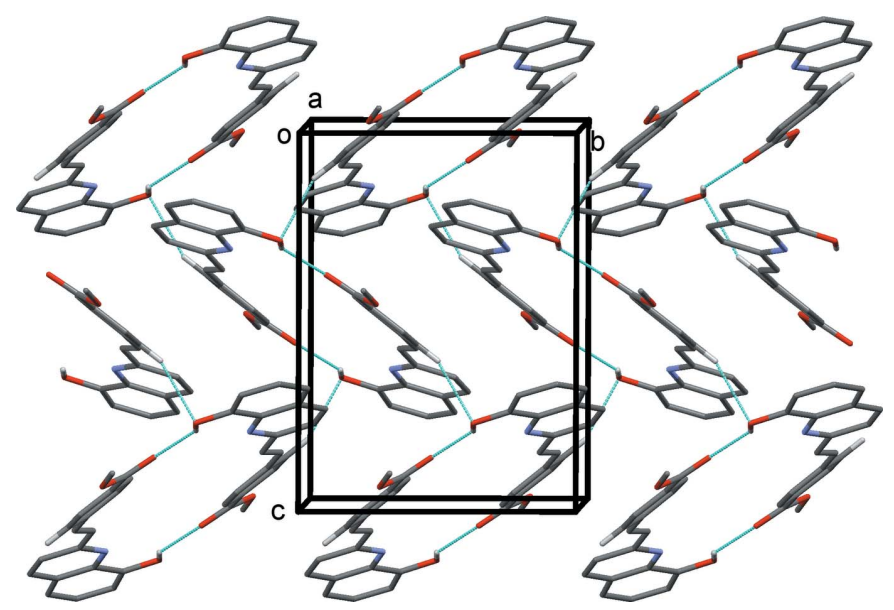

Figure 2

A view along the $a$ axis of the $R_{2}^{2}(28)$ ring motifs in the crystal of the title compound. Hydrogen bonds are shown as dashed lines (see Table 1), and for clarity only $\mathrm{H}$ atoms $\mathrm{H} 3 \mathrm{O}$ and $\mathrm{H} 5 \mathrm{~A}$ are included.
Table 1

Hydrogen-bond geometry $\left(\AA{ }^{\circ}\right)$.

$C g 1, C g 2$ and $C g 3$ are the centroids of rings N1/C11-C14/C19, C3-C8 and C14-C19, respectively.

\begin{tabular}{lllll}
\hline$D-\mathrm{H} \cdots A$ & $D-\mathrm{H}$ & $\mathrm{H} \cdots A$ & $D \cdots A$ & $D-\mathrm{H} \cdots A$ \\
\hline $\mathrm{O} 3-\mathrm{H} 3 O \cdots \mathrm{N} 1$ & $0.86(2)$ & $2.19(3)$ & $2.715(2)$ & $120(2)$ \\
$\mathrm{O} 3-\mathrm{H} 3 O \cdots 1^{\mathrm{i}}$ & $0.86(2)$ & $2.23(2)$ & $2.901(2)$ & $136(2)$ \\
$\mathrm{C} 5-\mathrm{H} 5 A \cdots{ }^{\mathrm{ii}}$ & 0.93 & 2.57 & $3.437(2)$ & 155 \\
$\mathrm{C} 7-\mathrm{H} 7 A \cdots C g 3^{\mathrm{iii}}$ & 0.93 & 2.99 & $3.605(2)$ & 125 \\
$\mathrm{C} 8-\mathrm{H} 8 A \cdots C g 1^{\mathrm{iii}}$ & 0.93 & 2.93 & $3.559(2)$ & 126 \\
$\mathrm{C} 15-\mathrm{H} 15 A \cdots C g 2^{\mathrm{ii}}$ & 0.93 & 2.83 & $3.639(2)$ & 146 \\
\hline
\end{tabular}

Symmetry codes: (i) $\quad-x+1,-y+1,-z ; \quad$ (ii) $\quad-x+\frac{3}{2}, y-\frac{1}{2},-z+\frac{1}{2}$; $\quad$ (iii) $x-\frac{1}{2},-y+\frac{1}{2}, z-\frac{1}{2}$.

compound in the sense that they also have an $E$ conformation about the $\mathrm{C}=\mathrm{C}$ bond, and in the crystal they also form inversion dimers. They include 2-\{2-[4-(trifluoromethyl)phenyl]vinyl\}quinolin-8-ol (HUKTOY; Huo et al., 2015), 2-[2-(4methoxyphenyl)vinyl]quinolin-8-ol (MIMPOP; Yuan et al., 2013), and 2-[2-(2,4-dinitrophenyl)vinyl]quinolin-8-ol (WELKEF; Yuan et al., 2013). In these three compounds, the quinoline and benzene rings are inclined to one another by $36.72(10)$ and $16.66(10)^{\circ}$ in HUKTOY (there are two independent molecules in the asymmetric unit), $42.59(7)^{\circ}$ in

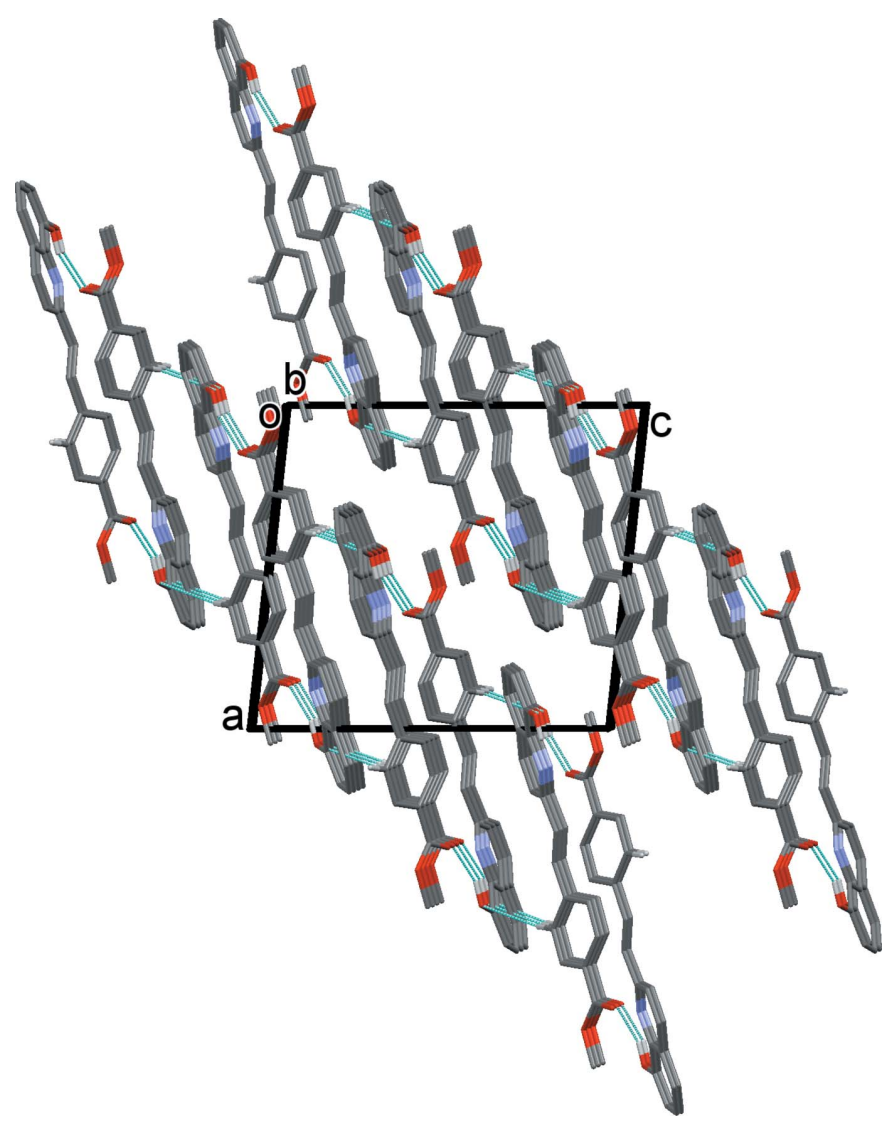

Figure 3

A view along the $b$ axis of the crystal packing of the title compound. Hydrogen bonds are shown as dashed lines (see Table 1) and, for clarity, only $\mathrm{H}$ atoms $\mathrm{H} 3 \mathrm{O}$ and $\mathrm{H} 5 \mathrm{~A}$ are included. 
Table 2

Experimental details.

\begin{tabular}{|c|c|}
\hline \multicolumn{2}{|l|}{ Crystal data } \\
\hline Chemical formula & $\mathrm{C}_{19} \mathrm{H}_{15} \mathrm{NO}_{3}$ \\
\hline$M_{\mathrm{r}}$ & 305.32 \\
\hline Crystal system, space group & Monoclinic, $P 2_{1} / n$ \\
\hline Temperature $(\mathrm{K})$ & 296 \\
\hline$a, b, c(\AA)$ & $12.0236(4), 9.7045$ (4), 13.2607 (4) \\
\hline$\beta\left(^{\circ}\right)$ & $96.260(2)$ \\
\hline$V\left(\AA^{3}\right)$ & $1538.07(9)$ \\
\hline$Z$ & 4 \\
\hline Radiation type & Мо $K \alpha$ \\
\hline$\mu\left(\mathrm{mm}^{-1}\right)$ & 0.09 \\
\hline Crystal size (mm) & $0.08 \times 0.06 \times 0.05$ \\
\hline \multicolumn{2}{|l|}{ Data collection } \\
\hline Diffractometer & $\begin{array}{l}\text { Bruker SMART CCD area- } \\
\text { detector }\end{array}$ \\
\hline Absorption correction & $\begin{array}{l}\text { Multi-scan (SADABS; Bruker, } \\
\text { 2005) }\end{array}$ \\
\hline$T_{\min }, T_{\max }$ & $0.993,0.996$ \\
\hline $\begin{array}{l}\text { No. of measured, independent and } \\
\text { observed }[I>2 \sigma(I)] \text { reflections }\end{array}$ & $13068,3511,2049$ \\
\hline$R_{\text {int }}$ & 0.041 \\
\hline$(\sin \theta / \lambda)_{\max }\left(\AA^{-1}\right)$ & 0.651 \\
\hline \multicolumn{2}{|l|}{ Refinement } \\
\hline$R\left[F^{2}>2 \sigma\left(F^{2}\right)\right], w R\left(F^{2}\right), S$ & $0.046,0.137,1.02$ \\
\hline No. of reflections & 3511 \\
\hline No. of parameters & 217 \\
\hline $\mathrm{H}$-atom treatment & $\begin{array}{l}\mathrm{H} \text { atoms treated by a mixture of } \\
\text { independent and constrained } \\
\text { refinement }\end{array}$ \\
\hline$\Delta \rho_{\max }, \Delta \rho_{\min }\left(\mathrm{e} \AA^{-3}\right)$ & $0.17,-0.18$ \\
\hline
\end{tabular}

Computer programs: SMART and SAINT (Bruker, 2005), SHELXS97, SHELXL97 and SHELXTL (Sheldrick, 2008), Mercury (Macrae et al., 2008) and PLATON (Spek, 2009).

MIMPOP and $5.63(6)^{\circ}$ in WELKEF, compared to $29.22(7)^{\circ}$ in the title compound.

\section{Synthesis and crystallization}

The title compound was prepared following reported procedures (Jing et al., 2006; Yuan et al., 2012). A mixture of 2-methy-8-hydroxyquinoline (1.59 g, $10 \mathrm{mmol})$, 4-formyl-2methylbenzoate $(1.64 \mathrm{~g}, 10 \mathrm{mmol})$ and acetic anhydride $(20 \mathrm{ml})$ was stirred for $12 \mathrm{~h}$ at $423 \mathrm{~K}$ under a nitrogen atmosphere. After cooling it was poured into ice-water $(150 \mathrm{ml})$ and stirred for $1-2 \mathrm{~h}$. Then, the puce solid obtained was filtered and together with triethylamine $(1 \mathrm{~g}, 10 \mathrm{mmol})$ was dissolved in DMF $(30 \mathrm{ml})$ and the mixture stirred for $3 \mathrm{~h}$ at
408 K. After cooling, the reaction mixture was concentrated and purified by chromatography on silica gel (petroleum ether/EtOAc $=3 / 1$ ). The product obtained was dissolved in ethanol, and on slow evaporation of the solvent yellow crystals were obtained within 2 weeks.

\section{Refinement}

Crystal data, data collection and structure refinement details are summarized in Table 2. The hydroxy-H atom was located in a difference Fourier map and freely refined. The C-bound $\mathrm{H}$ atoms were positioned geometrically and allowed to ride on their parent atoms: $\mathrm{C}-\mathrm{H}=0.93-0.96 \AA$ with $U_{\text {iso }}(\mathrm{H})=$ $1.5 U_{\text {eq }}(\mathrm{C}$-methyl $)$ and $1.2 U_{\text {eq }}(\mathrm{C})$ for other $\mathrm{H}$ atoms.

\section{Acknowledgements}

We gratefully acknowledge the support of the Natural Science Foundation of Zhejiang Province (LY12B01003).

\section{References}

Albrecht, M., Fiege, M. \& Osetska, O. (2008). Coord. Chem. Rev. 252, 812-824.

Bruker (2005). SMART, SAINT and SADABS. Bruker AXS Inc., Madison, Wisconsin, USA.

Cacciatore, I., Fornasari, E., Baldassare, L., Cornacchia, C., Fulle, S., DiFilippo, E. S., Pietrangelo, T. \& Pinnen, F. (2013). Pharmaceuticals, 6, 54-69.

Groom, C. R., Bruno, I. J., Lightfoot, M. P. \& Ward, S. C. (2016). Acta Cryst. B72, 171-179.

Huo, Y., Wang, C., Lu, J., Hu, S., Li, X. \& Zhang, L. (2015). J. Mol. Struct. 1098, 311-317.

Jing, H.-L., Zeng, H.-P., Zhou, Y.-D., Wang, T.-T., Yuan, G.-Z. \& Ouyang, X.-H. (2006). Chin. J. Chem. 24, 966-972.

Macrae, C. F., Bruno, I. J., Chisholm, J. A., Edgington, P. R., McCabe, P., Pidcock, E., Rodriguez-Monge, L., Taylor, R., van de Streek, J. \& Wood, P. A. (2008). J. Appl. Cryst. 41, 466-470.

Moulinet d'Hardemare, A. du, Gellon, G., Philouze, C. \& Serratrice, G. (2012). Inorg. Chem. 51, 12142-12151.

Sheldrick, G. M. (2008). Acta Cryst. A64, 112-122.

Song, K.-C., Kim, J.-S., Park, S.-M., Chung, K.-C., Ahn, S. \& Chang, S.-K. (2006). Org. Lett. 8, 3413-3416.

Spek, A. L. (2009). Acta Cryst. D65, 148-155.

Tang, C.-W. \& VanSlyke, S. A. (1987). Appl. Phys. Lett. 51, 913-915.

Yuan, G.-Z., Huo, Y.-P., Rong, L.-L., Nie, X.-L. \& Fang, X.-M. (2012). Inorg. Chem. Commun. 23, 90-94.

Yuan, G.-Z., Rong, L.-L., Qiao, X.-L., Xia, Y.-P., Guo, T. \& Wei, X.-W. (2013). Wuji Huaxue Xuebao, 29, 1769. 


\section{supporting information}

Acta Cryst. (2016). E72, 1251-1253 [https://doi.org/10.1107/S205698901601210X]

\section{Crystal structure of methyl $(E)-4-[2-(8-h y d r o x y q u i n o l i n-2-y l)$ vinyl]benzoate}

\section{Yu-Xing Xu, Wei-Ji Hu and Guo-Liang Zhao}

\section{Computing details}

Data collection: SMART (Bruker, 2005); cell refinement: SAINT (Bruker, 2005); data reduction: SAINT (Bruker, 2005); program(s) used to solve structure: SHELXS97 (Sheldrick, 2008); program(s) used to refine structure: SHELXL97 (Sheldrick, 2008); molecular graphics: SHELXTL (Sheldrick, 2008) and Mercury (Macrae et al., 2008); software used to prepare material for publication: SHELXL97 (Sheldrick, 2008) and PLATON (Spek, 2009).

Methyl (E)-4-[2-(8-hydroxyquinolin-2-yl)vinyl]benzoate

\section{Crystal data}

$\mathrm{C}_{19} \mathrm{H}_{15} \mathrm{NO}_{3}$

$M_{r}=305.32$

Monoclinic, $P 2_{1} / n$

Hall symbol: -P 2 yn

$a=12.0236(4) \AA$

$b=9.7045(4) \AA$

$c=13.2607(4) \AA$

$\beta=96.260(2)^{\circ}$

$V=1538.07(9) \AA^{3}$

$Z=4$

\section{Data collection}

Bruker SMART CCD area-detector diffractometer

Radiation source: fine-focus sealed tube Graphite monochromator

phi and $\omega$ scans

Absorption correction: multi-scan

(SADABS; Bruker, 2005)

$T_{\min }=0.993, T_{\max }=0.996$

\section{Refinement}

Refinement on $F^{2}$

Least-squares matrix: full

$R\left[F^{2}>2 \sigma\left(F^{2}\right)\right]=0.046$

$w R\left(F^{2}\right)=0.137$

$S=1.02$

3511 reflections

217 parameters

0 restraints

Primary atom site location: structure-invariant direct methods
$F(000)=640$

$D_{\mathrm{x}}=1.319 \mathrm{Mg} \mathrm{m}^{-3}$

Mo $K \alpha$ radiation, $\lambda=0.71073 \AA$

Cell parameters from 1747 reflections

$\theta=2.2-27.6^{\circ}$

$\mu=0.09 \mathrm{~mm}^{-1}$

$T=296 \mathrm{~K}$

Block, yellow

$0.08 \times 0.06 \times 0.05 \mathrm{~mm}$

13068 measured reflections

3511 independent reflections

2049 reflections with $I>2 \sigma(I)$

$R_{\text {int }}=0.041$

$\theta_{\max }=27.6^{\circ}, \theta_{\min }=2.2^{\circ}$

$h=-15 \rightarrow 14$

$k=-12 \rightarrow 12$

$l=-17 \rightarrow 17$

Secondary atom site location: difference Fourier map

Hydrogen site location: inferred from neighbouring sites

$\mathrm{H}$ atoms treated by a mixture of independent and constrained refinement

$w=1 /\left[\sigma^{2}\left(F_{\mathrm{o}}^{2}\right)+(0.0631 P)^{2}+0.1091 P\right]$ where $P=\left(F_{\mathrm{o}}^{2}+2 F_{\mathrm{c}}{ }^{2}\right) / 3$

$(\Delta / \sigma)_{\max }<0.001$

$\Delta \rho_{\max }=0.17 \mathrm{e} \AA^{-3}$

$\Delta \rho_{\min }=-0.18$ e $\AA^{-3}$ 


\section{Special details}

Geometry. All esds (except the esd in the dihedral angle between two 1.s. planes) are estimated using the full covariance matrix. The cell esds are taken into account individually in the estimation of esds in distances, angles and torsion angles; correlations between esds in cell parameters are only used when they are defined by crystal symmetry. An approximate (isotropic) treatment of cell esds is used for estimating esds involving l.s. planes.

Refinement. Refinement of $\mathrm{F}^{2}$ against ALL reflections. The weighted R-factor $\mathrm{wR}$ and goodness of fit $\mathrm{S}$ are based on $\mathrm{F}^{2}$, conventional $R$-factors $R$ are based on $F$, with $F$ set to zero for negative $F^{2}$. The threshold expression of $F^{2}>2 \operatorname{sigma}\left(\mathrm{F}^{2}\right)$ is used only for calculating R-factors(gt) etc. and is not relevant to the choice of reflections for refinement. R-factors based on $\mathrm{F}^{2}$ are statistically about twice as large as those based on F, and R- factors based on ALL data will be even larger.

Fractional atomic coordinates and isotropic or equivalent isotropic displacement parameters $\left(\hat{A}^{2}\right)$

\begin{tabular}{|c|c|c|c|c|}
\hline & $x$ & $y$ & $z$ & $U_{\text {iso }} * / U_{\text {eq }}$ \\
\hline N1 & $0.87623(11)$ & $0.22440(15)$ & $0.17162(11)$ & $0.0507(4)$ \\
\hline $\mathrm{O} 3$ & $1.04463(12)$ & $0.41136(15)$ & $0.20030(12)$ & $0.0678(4)$ \\
\hline C19 & $0.97735(12)$ & $0.17971(18)$ & $0.21670(12)$ & $0.0465(4)$ \\
\hline $\mathrm{C} 3$ & $0.26628(13)$ & $0.27214(18)$ & $-0.01100(13)$ & $0.0496(4)$ \\
\hline $\mathrm{C} 14$ & 0.99928 (13) & $0.04272(18)$ & $0.24806(12)$ & $0.0481(4)$ \\
\hline $\mathrm{C} 11$ & $0.79264(13)$ & $0.13449(18)$ & 0.15909 (12) & $0.0491(4)$ \\
\hline $\mathrm{O} 2$ & $0.07181(10)$ & $0.24711(14)$ & $-0.02756(10)$ & $0.0691(4)$ \\
\hline C6 & $0.47912(13)$ & $0.17659(19)$ & $0.06283(12)$ & 0.0508 (4) \\
\hline $\mathrm{C} 13$ & 0.90819 (14) & $-0.04932(19)$ & $0.23276(13)$ & $0.0544(5)$ \\
\hline $\mathrm{H} 13 \mathrm{~A}$ & 0.9178 & -0.1412 & 0.2517 & $0.065^{*}$ \\
\hline C18 & $1.06399(13)$ & $0.27912(19)$ & $0.23164(13)$ & $0.0521(4)$ \\
\hline $\mathrm{C} 12$ & 0.80665 (14) & $-0.0046(2)$ & $0.19061(13)$ & $0.0549(5)$ \\
\hline $\mathrm{H} 12 \mathrm{~A}$ & 0.7464 & -0.0651 & 0.1824 & $0.066^{*}$ \\
\hline $\mathrm{C} 2$ & $0.15483(14)$ & $0.3263(2)$ & $-0.05173(14)$ & $0.0574(5)$ \\
\hline $\mathrm{O} 1$ & $0.14069(11)$ & $0.42886(17)$ & $-0.10263(13)$ & $0.0939(5)$ \\
\hline $\mathrm{C} 10$ & $0.68606(13)$ & $0.18748(19)$ & $0.11050(13)$ & $0.0550(5)$ \\
\hline $\mathrm{H} 10 \mathrm{~A}$ & 0.6873 & 0.2733 & 0.0795 & $0.066^{*}$ \\
\hline C9 & $0.58827(13)$ & $0.1246(2)$ & $0.10668(13)$ & $0.0550(5)$ \\
\hline H9A & 0.5887 & 0.0370 & 0.1352 & $0.066^{*}$ \\
\hline $\mathrm{C} 8$ & $0.36132(14)$ & $0.3354(2)$ & $-0.03918(13)$ & $0.0587(5)$ \\
\hline H8A & 0.3541 & 0.4107 & -0.0828 & $0.070 *$ \\
\hline C15 & $1.10686(14)$ & $0.0073(2)$ & $0.29359(13)$ & $0.0581(5)$ \\
\hline $\mathrm{H} 15 \mathrm{~A}$ & 1.1226 & -0.0831 & 0.3136 & $0.070 *$ \\
\hline $\mathrm{C} 17$ & $1.16678(14)$ & $0.2423(2)$ & $0.27826(15)$ & $0.0611(5)$ \\
\hline H17A & 1.2229 & 0.3081 & 0.2901 & $0.073^{*}$ \\
\hline $\mathrm{C} 7$ & $0.46638(14)$ & $0.2885(2)$ & $-0.00345(14)$ & $0.0587(5)$ \\
\hline H7A & 0.5293 & 0.3318 & -0.0237 & $0.070^{*}$ \\
\hline $\mathrm{C} 4$ & $0.27801(14)$ & $0.15980(19)$ & $0.05325(14)$ & $0.0564(5)$ \\
\hline $\mathrm{H} 4 \mathrm{~A}$ & 0.2148 & 0.1156 & 0.0721 & $0.068^{*}$ \\
\hline C16 & $1.18752(15)$ & 0.1057 (2) & $0.30817(14)$ & $0.0641(5)$ \\
\hline H16A & 1.2581 & 0.0816 & 0.3387 & $0.077^{*}$ \\
\hline $\mathrm{C} 5$ & 0.38325 (14) & $0.11293(19)$ & 0.08960 (14) & $0.0589(5)$ \\
\hline $\mathrm{H} 5 \mathrm{~A}$ & 0.3900 & 0.0372 & 0.1329 & $0.071^{*}$ \\
\hline C1 & $-0.04060(15)$ & $0.2875(3)$ & $-0.06672(17)$ & $0.0812(7)$ \\
\hline H1B & -0.0932 & 0.2228 & -0.0445 & $0.122 *$ \\
\hline
\end{tabular}




\begin{tabular}{lllll}
$\mathrm{H} 1 \mathrm{C}$ & -0.0470 & 0.2886 & -0.1395 & $0.122^{*}$ \\
$\mathrm{H} 1 \mathrm{D}$ & -0.0561 & 0.3777 & -0.0421 & $0.122^{*}$ \\
$\mathrm{H} 3 \mathrm{O}$ & $0.9748(17)$ & $0.414(3)$ & $0.178(2)$ & $0.101(8)$ \\
\hline
\end{tabular}

Atomic displacement parameters $\left(\AA^{2}\right)$

\begin{tabular}{lllllll}
\hline & $U^{11}$ & $U^{22}$ & $U^{33}$ & $U^{12}$ & $U^{13}$ & $U^{23}$ \\
\hline $\mathrm{N} 1$ & $0.0405(7)$ & $0.0524(9)$ & $0.0578(9)$ & $0.0016(7)$ & $-0.0003(6)$ & $-0.0028(7)$ \\
$\mathrm{O} 3$ & $0.0509(8)$ & $0.0541(8)$ & $0.0935(10)$ & $-0.0052(7)$ & $-0.0140(8)$ & $0.0047(7)$ \\
$\mathrm{C} 19$ & $0.0397(9)$ & $0.0523(10)$ & $0.0470(9)$ & $0.0023(8)$ & $0.0028(7)$ & $-0.0005(8)$ \\
$\mathrm{C} 3$ & $0.0447(9)$ & $0.0557(11)$ & $0.0471(9)$ & $0.0034(8)$ & $-0.0015(7)$ & $-0.0011(8)$ \\
$\mathrm{C} 14$ & $0.0422(9)$ & $0.0528(11)$ & $0.0495(9)$ & $0.0062(8)$ & $0.0057(7)$ & $0.0010(8)$ \\
$\mathrm{C} 11$ & $0.0412(9)$ & $0.0503(10)$ & $0.0549(10)$ & $0.0016(8)$ & $0.0005(7)$ & $-0.0059(8)$ \\
$\mathrm{O} 2$ & $0.0414(7)$ & $0.0864(10)$ & $0.0774(9)$ & $0.0056(7)$ & $-0.0034(6)$ & $0.0080(7)$ \\
$\mathrm{C} 6$ & $0.0445(9)$ & $0.0530(10)$ & $0.0529(10)$ & $0.0011(8)$ & $-0.0034(7)$ & $-0.0051(8)$ \\
$\mathrm{C} 13$ & $0.0527(10)$ & $0.0471(10)$ & $0.0631(11)$ & $0.0035(8)$ & $0.0056(8)$ & $0.0009(8)$ \\
$\mathrm{C} 18$ & $0.0430(9)$ & $0.0531(11)$ & $0.0590(10)$ & $0.0007(8)$ & $0.0007(8)$ & $0.0001(9)$ \\
$\mathrm{C} 12$ & $0.0457(9)$ & $0.0515(11)$ & $0.0663(11)$ & $-0.0036(8)$ & $0.0013(8)$ & $-0.0061(9)$ \\
$\mathrm{C} 2$ & $0.0447(10)$ & $0.0687(13)$ & $0.0575(11)$ & $0.0050(10)$ & $0.0003(8)$ & $-0.0010(10)$ \\
$\mathrm{O} 1$ & $0.0597(9)$ & $0.0984(12)$ & $0.1208(13)$ & $0.0137(8)$ & $-0.0033(8)$ & $0.0450(11)$ \\
$\mathrm{C} 10$ & $0.0468(10)$ & $0.0530(11)$ & $0.0637(11)$ & $0.0046(8)$ & $-0.0011(8)$ & $-0.0015(9)$ \\
$\mathrm{C} 9$ & $0.0446(9)$ & $0.0549(11)$ & $0.0635(11)$ & $0.0026(8)$ & $-0.0032(8)$ & $-0.0027(9)$ \\
$\mathrm{C} 8$ & $0.0521(10)$ & $0.0638(12)$ & $0.0586(11)$ & $0.0015(9)$ & $-0.0004(8)$ & $0.0133(9)$ \\
$\mathrm{C} 15$ & $0.0456(10)$ & $0.0620(12)$ & $0.0661(11)$ & $0.0115(9)$ & $0.0034(8)$ & $0.0087(9)$ \\
$\mathrm{C} 17$ & $0.0424(10)$ & $0.0680(13)$ & $0.0713(12)$ & $-0.0049(9)$ & $-0.0011(8)$ & $-0.0012(10)$ \\
$\mathrm{C} 7$ & $0.0427(9)$ & $0.0703(13)$ & $0.0624(11)$ & $-0.0039(9)$ & $0.0027(8)$ & $0.0070(10)$ \\
$\mathrm{C} 4$ & $0.0436(9)$ & $0.0584(11)$ & $0.0657(11)$ & $-0.0046(8)$ & $-0.0005(8)$ & $0.0035(9)$ \\
$\mathrm{C} 16$ & $0.0404(9)$ & $0.0767(15)$ & $0.0731(12)$ & $0.0064(9)$ & $-0.0044(8)$ & $0.0075(11)$ \\
$\mathrm{C} 5$ & $0.0495(10)$ & $0.0554(11)$ & $0.0691(12)$ & $-0.0004(8)$ & $-0.0054(8)$ & $0.0110(9)$ \\
$\mathrm{C} 1$ & $0.0402(10)$ & $0.1128(19)$ & $0.0881(15)$ & $0.0112(11)$ & $-0.0039(9)$ & $0.0050(13)$ \\
$\mathrm{H} 3 \mathrm{O}$ & $0.039(13)$ & $0.12(2)$ & $0.14(2)$ & $-0.025(14)$ & $-0.010(13)$ & $0.009(16)$ \\
& & & & & & \\
\hline & & & & & &
\end{tabular}

Geometric parameters $\left(\AA,{ }^{o}\right)$

\begin{tabular}{llll}
\hline $\mathrm{N} 1-\mathrm{C} 11$ & $1.328(2)$ & $\mathrm{C} 18-\mathrm{C} 17$ & $1.368(2)$ \\
$\mathrm{N} 1-\mathrm{C} 19$ & $1.3657(19)$ & $\mathrm{C} 12-\mathrm{H} 12 \mathrm{~A}$ & 0.9300 \\
$\mathrm{O} 3-\mathrm{C} 18$ & $1.361(2)$ & $\mathrm{C} 2-\mathrm{O} 1$ & $1.204(2)$ \\
$\mathrm{O} 3-\mathrm{H} 3 \mathrm{O}$ & $0.86(2)$ & $\mathrm{C} 10-\mathrm{C} 9$ & $1.321(2)$ \\
$\mathrm{C} 19-\mathrm{C} 14$ & $1.409(2)$ & $\mathrm{C} 10-\mathrm{H} 10 \mathrm{~A}$ & 0.9300 \\
$\mathrm{C} 19-\mathrm{C} 18$ & $1.418(2)$ & $\mathrm{C} 9-\mathrm{H} 9 \mathrm{~A}$ & 0.9300 \\
$\mathrm{C} 3-\mathrm{C} 4$ & $1.381(2)$ & $\mathrm{C} 8-\mathrm{C} 7$ & $1.377(2)$ \\
$\mathrm{C} 3-\mathrm{C} 8$ & $1.384(2)$ & $\mathrm{C} 8-\mathrm{H} 8 \mathrm{~A}$ & 0.9300 \\
$\mathrm{C} 3-\mathrm{C} 2$ & $1.485(2)$ & $\mathrm{C} 15-\mathrm{C} 16$ & $1.360(3)$ \\
$\mathrm{C} 14-\mathrm{C} 15$ & $1.409(2)$ & $\mathrm{C} 15-\mathrm{H} 15 \mathrm{~A}$ & 0.9300 \\
$\mathrm{C} 14-\mathrm{C} 13$ & $1.411(2)$ & $\mathrm{C} 17-\mathrm{C} 16$ & $1.398(3)$ \\
$\mathrm{C} 11-\mathrm{C} 12$ & $1.417(3)$ & $\mathrm{C} 17-\mathrm{H} 17 \mathrm{~A}$ & 0.9300 \\
$\mathrm{C} 11-\mathrm{C} 10$ & $1.463(2)$ & $\mathrm{C} 7-\mathrm{H} 7 \mathrm{~A}$ & 0.9300 \\
$\mathrm{O} 2-\mathrm{C} 2$ & $1.326(2)$ & $\mathrm{C} 4-\mathrm{C} 5$ & $1.381(2)$
\end{tabular}




\begin{tabular}{|c|c|c|c|}
\hline $\mathrm{O} 2-\mathrm{C} 1$ & $1.448(2)$ & $\mathrm{C} 4-\mathrm{H} 4 \mathrm{~A}$ & 0.9300 \\
\hline $\mathrm{C} 6-\mathrm{C} 5$ & $1.388(2)$ & $\mathrm{C} 16-\mathrm{H} 16 \mathrm{~A}$ & 0.9300 \\
\hline $\mathrm{C} 6-\mathrm{C} 7$ & $1.395(3)$ & $\mathrm{C} 5-\mathrm{H} 5 \mathrm{~A}$ & 0.9300 \\
\hline $\mathrm{C} 6-\mathrm{C} 9$ & $1.466(2)$ & $\mathrm{C} 1-\mathrm{H} 1 \mathrm{~B}$ & 0.9600 \\
\hline $\mathrm{C} 13-\mathrm{C} 12$ & $1.357(2)$ & $\mathrm{C} 1-\mathrm{H} 1 \mathrm{C}$ & 0.9600 \\
\hline $\mathrm{C} 13-\mathrm{H} 13 \mathrm{~A}$ & 0.9300 & $\mathrm{C} 1-\mathrm{H} 1 \mathrm{D}$ & 0.9600 \\
\hline $\mathrm{C} 11-\mathrm{N} 1-\mathrm{C} 19$ & $118.15(15)$ & $\mathrm{C} 11-\mathrm{C} 10-\mathrm{H} 10 \mathrm{~A}$ & 117.0 \\
\hline $\mathrm{C} 18-\mathrm{O} 3-\mathrm{H} 3 \mathrm{O}$ & $105.2(17)$ & $\mathrm{C} 10-\mathrm{C} 9-\mathrm{C} 6$ & $127.66(18)$ \\
\hline $\mathrm{N} 1-\mathrm{C} 19-\mathrm{C} 14$ & $123.90(15)$ & $\mathrm{C} 10-\mathrm{C} 9-\mathrm{H} 9 \mathrm{~A}$ & 116.2 \\
\hline $\mathrm{N} 1-\mathrm{C} 19-\mathrm{C} 18$ & $116.78(16)$ & C6-C9-H9A & 116.2 \\
\hline $\mathrm{C} 14-\mathrm{C} 19-\mathrm{C} 18$ & $119.32(14)$ & $\mathrm{C} 7-\mathrm{C} 8-\mathrm{C} 3$ & $120.90(17)$ \\
\hline $\mathrm{C} 4-\mathrm{C} 3-\mathrm{C} 8$ & $119.02(15)$ & $\mathrm{C} 7-\mathrm{C} 8-\mathrm{H} 8 \mathrm{~A}$ & 119.5 \\
\hline $\mathrm{C} 4-\mathrm{C} 3-\mathrm{C} 2$ & $122.07(17)$ & $\mathrm{C} 3-\mathrm{C} 8-\mathrm{H} 8 \mathrm{~A}$ & 119.5 \\
\hline $\mathrm{C} 8-\mathrm{C} 3-\mathrm{C} 2$ & $118.91(17)$ & $\mathrm{C} 16-\mathrm{C} 15-\mathrm{C} 14$ & $119.79(18)$ \\
\hline $\mathrm{C} 15-\mathrm{C} 14-\mathrm{C} 19$ & $119.34(16)$ & $\mathrm{C} 16-\mathrm{C} 15-\mathrm{H} 15 \mathrm{~A}$ & 120.1 \\
\hline $\mathrm{C} 15-\mathrm{C} 14-\mathrm{C} 13$ & $124.70(17)$ & $\mathrm{C} 14-\mathrm{C} 15-\mathrm{H} 15 \mathrm{~A}$ & 120.1 \\
\hline $\mathrm{C} 19-\mathrm{C} 14-\mathrm{C} 13$ & $115.94(14)$ & $\mathrm{C} 18-\mathrm{C} 17-\mathrm{C} 16$ & $120.02(17)$ \\
\hline $\mathrm{N} 1-\mathrm{C} 11-\mathrm{C} 12$ & $121.65(14)$ & $\mathrm{C} 18-\mathrm{C} 17-\mathrm{H} 17 \mathrm{~A}$ & 120.0 \\
\hline $\mathrm{N} 1-\mathrm{C} 11-\mathrm{C} 10$ & $116.03(16)$ & $\mathrm{C} 16-\mathrm{C} 17-\mathrm{H} 17 \mathrm{~A}$ & 120.0 \\
\hline $\mathrm{C} 12-\mathrm{C} 11-\mathrm{C} 10$ & $122.32(15)$ & $\mathrm{C} 8-\mathrm{C} 7-\mathrm{C} 6$ & $120.51(17)$ \\
\hline $\mathrm{C} 2-\mathrm{O} 2-\mathrm{C} 1$ & $117.01(16)$ & $\mathrm{C} 8-\mathrm{C} 7-\mathrm{H} 7 \mathrm{~A}$ & 119.7 \\
\hline $\mathrm{C} 5-\mathrm{C} 6-\mathrm{C} 7$ & $118.07(15)$ & $\mathrm{C} 6-\mathrm{C} 7-\mathrm{H} 7 \mathrm{~A}$ & 119.7 \\
\hline $\mathrm{C} 5-\mathrm{C} 6-\mathrm{C} 9$ & $118.56(17)$ & $\mathrm{C} 5-\mathrm{C} 4-\mathrm{C} 3$ & $120.21(17)$ \\
\hline $\mathrm{C} 7-\mathrm{C} 6-\mathrm{C} 9$ & $123.36(17)$ & $\mathrm{C} 5-\mathrm{C} 4-\mathrm{H} 4 \mathrm{~A}$ & 119.9 \\
\hline $\mathrm{C} 12-\mathrm{C} 13-\mathrm{C} 14$ & $120.40(17)$ & $\mathrm{C} 3-\mathrm{C} 4-\mathrm{H} 4 \mathrm{~A}$ & 119.9 \\
\hline $\mathrm{C} 12-\mathrm{C} 13-\mathrm{H} 13 \mathrm{~A}$ & 119.8 & $\mathrm{C} 15-\mathrm{C} 16-\mathrm{C} 17$ & $121.54(16)$ \\
\hline $\mathrm{C} 14-\mathrm{C} 13-\mathrm{H} 13 \mathrm{~A}$ & 119.8 & $\mathrm{C} 15-\mathrm{C} 16-\mathrm{H} 16 \mathrm{~A}$ & 119.2 \\
\hline $\mathrm{O} 3-\mathrm{C} 18-\mathrm{C} 17$ & $120.02(17)$ & $\mathrm{C} 17-\mathrm{C} 16-\mathrm{H} 16 \mathrm{~A}$ & 119.2 \\
\hline $\mathrm{O} 3-\mathrm{C} 18-\mathrm{C} 19$ & $120.01(14)$ & $\mathrm{C} 4-\mathrm{C} 5-\mathrm{C} 6$ & $121.28(17)$ \\
\hline $\mathrm{C} 17-\mathrm{C} 18-\mathrm{C} 19$ & $119.96(17)$ & $\mathrm{C} 4-\mathrm{C} 5-\mathrm{H} 5 \mathrm{~A}$ & 119.4 \\
\hline $\mathrm{C} 13-\mathrm{C} 12-\mathrm{C} 11$ & $119.92(16)$ & $\mathrm{C} 6-\mathrm{C} 5-\mathrm{H} 5 \mathrm{~A}$ & 119.4 \\
\hline $\mathrm{C} 13-\mathrm{C} 12-\mathrm{H} 12 \mathrm{~A}$ & 120.0 & $\mathrm{O} 2-\mathrm{C} 1-\mathrm{H} 1 \mathrm{~B}$ & 109.5 \\
\hline $\mathrm{C} 11-\mathrm{C} 12-\mathrm{H} 12 \mathrm{~A}$ & 120.0 & $\mathrm{O} 2-\mathrm{C} 1-\mathrm{H} 1 \mathrm{C}$ & 109.5 \\
\hline $\mathrm{O} 1-\mathrm{C} 2-\mathrm{O} 2$ & $123.39(16)$ & $\mathrm{H} 1 \mathrm{~B}-\mathrm{C} 1-\mathrm{H} 1 \mathrm{C}$ & 109.5 \\
\hline $\mathrm{O} 1-\mathrm{C} 2-\mathrm{C} 3$ & $124.26(18)$ & $\mathrm{O} 2-\mathrm{C} 1-\mathrm{H} 1 \mathrm{D}$ & 109.5 \\
\hline $\mathrm{O} 2-\mathrm{C} 2-\mathrm{C} 3$ & $112.33(17)$ & $\mathrm{H} 1 \mathrm{~B}-\mathrm{C} 1-\mathrm{H} 1 \mathrm{D}$ & 109.5 \\
\hline $\mathrm{C} 9-\mathrm{C} 10-\mathrm{C} 11$ & $126.00(18)$ & $\mathrm{H} 1 \mathrm{C}-\mathrm{C} 1-\mathrm{H} 1 \mathrm{D}$ & 109.5 \\
\hline $\mathrm{C} 9-\mathrm{C} 10-\mathrm{H} 10 \mathrm{~A}$ & 117.0 & & \\
\hline $\mathrm{C} 11-\mathrm{N} 1-\mathrm{C} 19-\mathrm{C} 14$ & $2.0(2)$ & $\mathrm{C} 8-\mathrm{C} 3-\mathrm{C} 2-\mathrm{O} 2$ & $173.08(16)$ \\
\hline $\mathrm{C} 11-\mathrm{N} 1-\mathrm{C} 19-\mathrm{C} 18$ & $-178.23(15)$ & $\mathrm{N} 1-\mathrm{C} 11-\mathrm{C} 10-\mathrm{C} 9$ & $-167.28(17)$ \\
\hline $\mathrm{N} 1-\mathrm{C} 19-\mathrm{C} 14-\mathrm{C} 15$ & $179.78(15)$ & $\mathrm{C} 12-\mathrm{C} 11-\mathrm{C} 10-\mathrm{C} 9$ & $13.1(3)$ \\
\hline $\mathrm{C} 18-\mathrm{C} 19-\mathrm{C} 14-\mathrm{C} 15$ & $0.0(2)$ & $\mathrm{C} 11-\mathrm{C} 10-\mathrm{C} 9-\mathrm{C} 6$ & $177.07(16)$ \\
\hline $\mathrm{N} 1-\mathrm{C} 19-\mathrm{C} 14-\mathrm{C} 13$ & $-1.6(2)$ & $\mathrm{C} 5-\mathrm{C} 6-\mathrm{C} 9-\mathrm{C} 10$ & $-162.92(18)$ \\
\hline $\mathrm{C} 18-\mathrm{C} 19-\mathrm{C} 14-\mathrm{C} 13$ & $178.58(15)$ & $\mathrm{C} 7-\mathrm{C} 6-\mathrm{C} 9-\mathrm{C} 10$ & $16.0(3)$ \\
\hline $\mathrm{C} 19-\mathrm{N} 1-\mathrm{C} 11-\mathrm{C} 12$ & $-0.4(2)$ & $\mathrm{C} 4-\mathrm{C} 3-\mathrm{C} 8-\mathrm{C} 7$ & $-0.5(3)$ \\
\hline $\mathrm{C} 19-\mathrm{N} 1-\mathrm{C} 11-\mathrm{C} 10$ & $179.96(14)$ & $\mathrm{C} 2-\mathrm{C} 3-\mathrm{C} 8-\mathrm{C} 7$ & $-179.86(16)$ \\
\hline
\end{tabular}




\begin{tabular}{llll}
$\mathrm{C} 15-\mathrm{C} 14-\mathrm{C} 13-\mathrm{C} 12$ & $178.19(17)$ & $\mathrm{C} 19-\mathrm{C} 14-\mathrm{C} 15-\mathrm{C} 16$ & $1.0(3)$ \\
$\mathrm{C} 19-\mathrm{C} 14-\mathrm{C} 13-\mathrm{C} 12$ & $-0.3(2)$ & $\mathrm{C} 13-\mathrm{C} 14-\mathrm{C} 15-\mathrm{C} 16$ & $-177.45(18)$ \\
$\mathrm{N} 1-\mathrm{C} 19-\mathrm{C} 18-\mathrm{O} 3$ & $-0.9(2)$ & $\mathrm{O} 3-\mathrm{C} 18-\mathrm{C} 17-\mathrm{C} 16$ & $-178.39(18)$ \\
$\mathrm{C} 14-\mathrm{C} 19-\mathrm{C} 18-\mathrm{O} 3$ & $178.95(16)$ & $\mathrm{C} 19-\mathrm{C} 18-\mathrm{C} 17-\mathrm{C} 16$ & $2.1(3)$ \\
$\mathrm{N} 1-\mathrm{C} 19-\mathrm{C} 18-\mathrm{C} 17$ & $178.64(16)$ & $\mathrm{C} 3-\mathrm{C} 8-\mathrm{C} 7-\mathrm{C} 6$ & $-0.6(3)$ \\
$\mathrm{C} 14-\mathrm{C} 19-\mathrm{C} 18-\mathrm{C} 17$ & $-1.6(2)$ & $\mathrm{C} 5-\mathrm{C} 6-\mathrm{C} 7-\mathrm{C} 8$ & $1.3(3)$ \\
$\mathrm{C} 14-\mathrm{C} 13-\mathrm{C} 12-\mathrm{C} 11$ & $1.8(3)$ & $\mathrm{C} 9-\mathrm{C} 6-\mathrm{C} 7-\mathrm{C} 8$ & $-177.65(17)$ \\
$\mathrm{N} 1-\mathrm{C} 11-\mathrm{C} 12-\mathrm{C} 13$ & $-1.5(3)$ & $\mathrm{C} 8-\mathrm{C} 3-\mathrm{C} 4-\mathrm{C} 5$ & $0.8(3)$ \\
$\mathrm{C} 10-\mathrm{C} 11-\mathrm{C} 12-\mathrm{C} 13$ & $178.16(16)$ & $\mathrm{C} 2-\mathrm{C} 3-\mathrm{C} 4-\mathrm{C} 5$ & $-179.85(16)$ \\
$\mathrm{C} 1-\mathrm{O} 2-\mathrm{C} 2-\mathrm{O} 1$ & $0.9(3)$ & $\mathrm{C} 14-\mathrm{C} 15-\mathrm{C} 16-\mathrm{C} 17$ & $-0.5(3)$ \\
$\mathrm{C} 1-\mathrm{O} 2-\mathrm{C} 2-\mathrm{C} 3$ & $-177.71(15)$ & $\mathrm{C} 18-\mathrm{C} 17-\mathrm{C} 16-\mathrm{C} 15$ & $-1.1(3)$ \\
$\mathrm{C} 4-\mathrm{C} 3-\mathrm{C} 2-\mathrm{O} 1$ & $175.11(19)$ & $\mathrm{C} 3-\mathrm{C} 4-\mathrm{C} 5-\mathrm{C} 6$ & $-0.1(3)$ \\
$\mathrm{C} 8-\mathrm{C} 3-\mathrm{C} 2-\mathrm{O} 1$ & $-5.6(3)$ & $\mathrm{C} 7-\mathrm{C} 6-\mathrm{C} 5-\mathrm{C} 4$ & $-1.0(3)$ \\
$\mathrm{C} 4-\mathrm{C} 3-\mathrm{C} 2-\mathrm{O} 2$ & $-6.3(3)$ & $\mathrm{C} 9-\mathrm{C} 6-\mathrm{C} 5-\mathrm{C} 4$ & $178.01(16)$ \\
\hline
\end{tabular}

Hydrogen-bond geometry $\left(\AA,{ }^{\circ}\right)$

$C g 1, C g 2$ and $C g 3$ are the centroids of rings $\mathrm{N1} / \mathrm{C} 11-\mathrm{C} 14 / \mathrm{C} 19, \mathrm{C} 3-\mathrm{C} 8$ and $\mathrm{C} 14-\mathrm{C} 19$, respectively.

\begin{tabular}{lllll}
\hline$D-\mathrm{H} \cdots A$ & $D-\mathrm{H}$ & $\mathrm{H} \cdots A$ & $D \cdots A$ & $D-\mathrm{H}^{\cdots} A$ \\
\hline $\mathrm{O} 3-\mathrm{H} 3 O \cdots \mathrm{N} 1$ & $0.86(2)$ & $2.19(3)$ & $2.715(2)$ & $120(2)$ \\
$\mathrm{O} 3-\mathrm{H} 3 O \cdots \mathrm{O} 1^{\mathrm{i}}$ & $0.86(2)$ & $2.23(2)$ & $2.901(2)$ & $136(2)$ \\
$\mathrm{C} 5-\mathrm{H} 5 A \cdots \mathrm{O} 3^{\mathrm{ii}}$ & 0.93 & 2.57 & $3.437(2)$ & 155 \\
$\mathrm{C} 7-\mathrm{H} 7 A \cdots C g 3^{\mathrm{iii}}$ & 0.93 & 2.99 & $3.605(2)$ & 125 \\
$\mathrm{C} 8-\mathrm{H} 8 A \cdots C g 1^{\mathrm{iii}}$ & 0.93 & 2.93 & $3.559(2)$ & 126 \\
$\mathrm{C} 15-\mathrm{H} 15 A \cdots C g 2^{\mathrm{ii}}$ & 0.93 & 2.83 & $3.639(2)$ & 146 \\
\hline
\end{tabular}

Symmetry codes: (i) $-x+1,-y+1,-z$; (ii) $-x+3 / 2, y-1 / 2,-z+1 / 2$; (iii) $x-1 / 2,-y+1 / 2, z-1 / 2$. 